\title{
Unscented Kalman filters for estimating the position of an automotive electronic throttle valve
}

\author{
Alessandro N. Vargas, Henrique M. T. Menegaz, João Y. Ishihara, \\ and Leonardo Acho
}

\begin{abstract}
The paper presents an application of unscented Kalman filters to an automotive electronic throttle device. The motivation of this study is on estimating the position of the throttle device when measurements of position are inaccessible, e.g., due to failures in the sensor of position. In this case, an external wattmeter is connected in the circuitry to measure the power consumed by the throttle, and this information feeds unscented Kalman filters to produce the estimation for the position. Experimental data support the findings of this paper.

Note to Practitioners: Abstract-Almost all of the brand-new vehicles based on spark-ignition combustion engines have an electronic throttle valve to control the power produced by the engine. The electronic throttle has a unique sensor for measuring the position of the throttle valve, and this feature can represent a serious problem when the sensor of position fails. As an attempt to prevent the effects of a failure from such sensor, we present an algorithm (unscented Kalman filter) combined with the use of an additional sensor, a wattmeter. The wattmeter is detached from the throttle's structure but is arranged so as to measure the electric power consumed by the throttle. Measurements of the power consumption then feed the unscented Kalman filter-this filter then produces an estimation of the position of the throttle valve. Experimental data illustrate the practical benefits of our approach.
\end{abstract}

Index Terms-Stochastic systems; Nonlinear systems; Switching systems; Unscented Kalman filter; automotive applications.

\section{INTRODUCTION}

An innovative technology that has been intensively improved by the automotive's industry in the last few years is the electronic throttle body. Made up by a circular plate moving around a central axis, the throttle body is a fundamental mechanism used in almost all modern spark-ignition combustion engines. The throttle's task is to regulate the power produced by the engine, and to do so, the throttle controls the amount of air entering into the combustion chambers. The rich literature has confirmed the importance of improving the throttle's functionality, see for instance [1]-[9] for a brief account.

Copyright (c) 2015 IEEE. Personal use of this material is permitted. However, permission to use this material for any other purposes must be obtained from the IEEE by sending a request to pubs-permissions@ieee.org. Research supported in part by the Brazilian agencies CNPq Grants 304856/20070, 312627/2013-0, and 248470/2013-2; and CAPES Grant Programa PVE 88881.030423/2013-01.

A. N. Vargas is with Universidade Tecnológica Federal do Paraná, UTFPR, Av. Alberto Carazzai 1640, 86300-000, Cornélio Procópio, PR, Brazil; H. M. T. Menegaz and J. Y. Ishihara are with Automation and Robotics Laboratory, University of Brasília, Brasília, DF 70910-900 Brazil; and L. Acho is with CoDAlab (Control, Dynamics and Applications), Departament de Matemàtica Aplicada III, Universitat Politècnica de Catalunya, Comte d'Urgell, 187, 08036 Barcelona, Spain. E-mail: avargas eutfpr.edu.br.

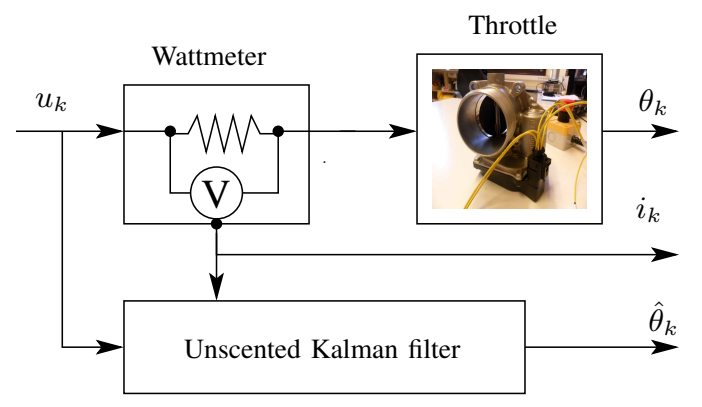

Fig. 1. Diagram of the input-output relationship for an automotive electronic throttle device implemented in a laboratory testbed. A wattmeter was added in series with the throttle circuit to measure the electrical current consumed by it (variable $i_{k}$ ). The real-time position of the throttle (see model in (2)) and its estimation from the unscented Kalman filter (see Algorithms in Section II) are denoted by $\theta_{k}$ and $\hat{\theta}_{k}$, respectively. The voltage input is denoted by $u_{k}$.

The throttle is a single-input single-output process. When a voltage is applied in its input, the apparatus generates an angular movement of the throttle valve; and a sensor measures the angular position of the valve.

Even though reliable and vastly used by the automotive industry, the sensor of position is not free of failures at all. In case of failure, the throttle's functionality becomes deteriorated, a fact that increases the risks of damage - some specialists argue that the sudden acceleration in Toyota's vehicles are related to failures in the throttle [10, p. 478-479]. Also, failures in the throttle's functionality may appear due to tin whiskers [11], [12]. In summary, failures in the throttle's functionality are unacceptable.

Our main idea to overcome the effects of a failure in the sensor of position is to add in the circuitry a new sensor. This new sensor is detached from the throttle's body, but it is positioned in series with the throttle's input so as to measure the power consumed by the throttle. The measurement from the new sensor then feeds unscented Kalman filters, and the filters estimate the position of the throttle-notice that the filters rely only on the measurements from this new sensor (Fig. 1). Although simple, our idea is motivated by the fact that both the position and electrical current represent system states in the throttle's model, an intricate nonlinear model [7], [13], [14]. Estimating the position of the throttle through unscented Kalman filters sets the main finding of this paper.

Unscented Kalman filters have drawn the attention of many investigators due to its superior performance when compared with other type of filters [15]-[22], with many applications, such as on electroencephalographies [23], plasma insulins [24], electronic battery charging [25], aeronautics [26], [27], 
monocular visual simultaneous localization and mapping [28], among others.

Besides these interesting applications, unscented Kalman filters are also useful to processes with failures in sensors. For instance, in this paper unscented Kalman filters are used to estimate the position of an automotive throttle valve with no sensor of position at all. Experimental data suggested that the estimation was quite accurate, with an error of $0.7^{\circ} \pm 13.4^{\circ}$ (confidence level of 95\%). This confirms the practical implications of our approach.

The paper is organized as follows. Section II quotes the basic notions and presents the filters. Section III presents experimental results that illustrate the usefulness of the filters. Finally, Section IV presents some concluding remarks.

\section{The STOCHAStiC SYSTEM AND UnSCENTED KALMAN FILTERS}

As usual, $\mathbb{R}^{n}$ denotes the $n$-dimensional Euclidean space, and $\mathbb{R}^{n \times m}$ denotes the set of matrices of dimension $n \times m$. For a matrix $A, A^{\prime}$ stands for its transpose, $(A)_{j i}$ for its $j$-th row and $i$-th column element, $(A)_{* i}$ for its $i$-th column, and $[A]_{p \times q}$ for a block matrix made up by $A$ being repeated $p$ times on the rows and $q$ times on the columns.

Let $(\Omega, \mathcal{F}, P)$ be a fixed probability space governing the following discrete-time, stochastic nonlinear system:

$$
\mathcal{S}:\left\{\begin{array}{l}
x_{k+1}=f\left(x_{k}\right)+F w_{k}, \\
y_{k}=h\left(x_{k}\right)+H v_{k}, \quad \forall k \geq 0, \quad x_{0}=x \in \mathbb{R}^{n},
\end{array}\right.
$$

where $x_{k}$ on $\mathbb{R}^{n}$ denotes the system state and $y_{k}$ on $\mathbb{R}^{m}$ represents the measured output; both $\left\{w_{k}\right\}$ on $\mathbb{R}^{n}$ and $\left\{v_{k}\right\}$ on $\mathbb{R}^{m}$ denote standard Gaussian noise sequences; and the matrices $F \in \mathbb{R}^{n \times n}$ and $H \in \mathbb{R}^{m \times m}$ are given. The functions $f: \mathbb{R}^{n} \rightarrow \mathbb{R}^{n}$ and $h: \mathbb{R}^{n} \rightarrow \mathbb{R}^{m}$ are Borel measurable.

The filtering problem associated with $\mathcal{S}$ is that of finding an estimate $\hat{x}_{k}$ for the system state $x_{k}$ as long as new measurements $y_{k}$ are acquired. This signifies that the output history until the $k$-th stage, i.e., $\left(y_{0}, \ldots, y_{k}\right)$, is used by the filter to compute $\hat{x}_{k}$; hopefully, the estimated value in $\hat{x}_{k}$ is close enough to the real value $x_{k}$. The manner for which unscented Kalman filters estimate $\hat{x}_{k}$ is now clarified.

\section{A. Algorithms for unscented Kalman filters}

We wish to emphasize here the similarities and differences found in different versions of unscented Kalman filters. For this reason, we first recall the version referred to as Additive Homogeneous Symmetric Minimum UKF [15] (see also [19]).

Additive Homogeneous Symmetric Minimum (AHSM) UKF

- Step 1: Set $N=2 n$, and take the initial values for $\hat{x}_{0 \mid 0} \in$ $\mathbb{R}^{n}$ and for a positive semidefinite matrix $\hat{P}_{x x}^{0 \mid 0} \in \mathbb{R}^{n \times n}$. Pick an arbitrary value $\lambda \neq-n$, and set $w_{0}=\lambda /(n+\lambda)$ and $w_{i}=w_{i+n}=1 / 2(n+\lambda), i=1, \ldots, n$. Set the iterations counter $k=1$ and go to the next step.
- Step 2: Take $\chi_{0}^{k-1 \mid k-1}=\hat{x}_{k-1 \mid k-1}$, and compute

$$
\begin{aligned}
& \chi_{i}^{k-1 \mid k-1}=\hat{x}_{k-1 \mid k-1}+\left(\sqrt{(n+\lambda) \hat{P}_{x x}^{k-1 \mid k-1}}\right)_{* i}, \\
& \text { and } \quad \chi_{i+n}^{k-1 \mid k-1}=\hat{x}_{k-1 \mid k-1}-\left(\sqrt{(n+\lambda) \hat{P}_{x x}^{k-1 \mid k-1}}\right)_{* i}, \\
& i=1, \ldots, n .
\end{aligned}
$$

Go to Step 3.

- Step 3: Compute

$$
\begin{aligned}
& \chi_{i}^{k \mid k-1}=f\left(\chi_{i}^{k-1 \mid k-1}\right), \\
& \gamma_{i}^{k \mid k-1}=h\left(\chi_{i}^{k \mid k-1}\right), \quad i=0, \ldots, N,
\end{aligned}
$$

and evaluate the vectors

$$
\hat{x}_{k \mid k-1}=\sum_{s=0}^{N} w_{i} \chi_{i}^{k \mid k-1}, \quad \hat{y}_{k \mid k-1}=\sum_{s=0}^{N} w_{i} \gamma_{i}^{k \mid k-1},
$$

and the matrices

$$
\begin{aligned}
\hat{P}_{x x}^{k \mid k-1}= & \sum_{s=0}^{N} w_{i}\left(\chi_{i}^{k \mid k-1}-\hat{x}_{k \mid k-1}\right)\left(\chi_{i}^{k \mid k-1}-\hat{x}_{k \mid k-1}\right)^{\prime} \\
& +F F^{\prime}, \\
\hat{P}_{x y}^{k \mid k-1}= & \sum_{s=0}^{N} w_{i}\left(\chi_{i}^{k \mid k-1}-\hat{x}_{k \mid k-1}\right)\left(\gamma_{i}^{k \mid k-1}-\hat{y}_{k \mid k-1}\right)^{\prime} \\
\hat{P}_{y y}^{k \mid k-1}= & \sum_{s=0}^{N} w_{i}\left(\gamma_{i}^{k \mid k-1}-\hat{y}_{k \mid k-1}\right)\left(\gamma_{i}^{k \mid k-1}-\hat{y}_{k \mid k-1}\right)^{\prime} \\
& +H H^{\prime} .
\end{aligned}
$$

Compute the matrices $G_{k}=\hat{P}_{x y}^{k \mid k-1}\left(\hat{P}_{y y}^{k \mid k-1}\right)^{-1}$,

$$
\begin{aligned}
& \hat{x}_{k \mid k}=\hat{x}_{k \mid k-1}+G_{k}\left(y_{k}-\hat{y}_{k \mid k-1}\right) \\
& \text { and } \hat{P}_{x x}^{k \mid k}=\hat{P}_{x x}^{k \mid k-1}-G_{k} \hat{P}_{y y}^{k \mid k-1} G_{k}^{\prime} .
\end{aligned}
$$

Set $k=k+1$ and return to the beginning of Step 2 .

Other filter that produces promising results is referred to as Additive Rho Minimum (ARM) UKF [29], and it is obtained by modifying Step 1 and Step 2 according to the next procedure:

- Step 1a: Set $N=n$, and take the initial values for $\hat{x}_{0 \mid 0} \in$ $\mathbb{R}^{n}$ and for a positive semidefinite matrix $\hat{P}_{x x}^{0 \mid 0} \in \mathbb{R}^{n \times n}$. Pick an arbitrary value $0<w_{0}<1$ and set $\rho=\sqrt{\frac{1-w_{0}}{n}}$; $C=\sqrt{I_{n}-\rho^{2}[1]_{n \times n}}$; and $W=\operatorname{diag}\left(w_{1}, \ldots, w_{n}\right)$, where

$$
w_{i}=\left(w_{0} \rho^{2} C^{-1}[1]_{n \times n}\left(C^{T}\right)^{-1}\right)_{i i}, \quad i=1, \ldots, n .
$$

Set the iterations counter $k=1$ and go to the next step.

- Step 2a: Compute the equation (1) of the next page. Go to Step 3 and follow it completely, but with its last command referring to Step $2 a$ instead of Step 2.

Other promising filter available in the literature is known as Additive General Minimum (AGM) UKF [18], which reads as follows:

- Step 1b: Set $N=n$, and take the initial values for $\hat{x}_{0 \mid 0} \in$ $\mathbb{R}^{n}$ and for a positive semidefinite matrix $\hat{P}_{x x}^{0 \mid 0} \in \mathbb{R}^{n \times n}$. Pick an arbitrary value $v:=\left[v_{1} \ldots v_{n}\right]^{T} \in \mathbb{R}^{n}, v_{i} \neq 0$, 


$$
\chi_{i}^{k-1 \mid k-1}=\hat{x}_{k-1 \mid k-1}+\left(\left[\frac{-1}{\sqrt{w_{0}}} \sqrt{\hat{P}_{x x}^{k-1 \mid k-1}}[\rho]_{n \times 1} \quad \sqrt{\hat{P}_{x x}^{k-1 \mid k-1}} C(\sqrt{W})^{-1}\right]\right)_{* i}, i=0, \ldots, n
$$

$i=1, \ldots, n$, and set $w_{0}=\left(1+\sum_{i=1}^{n} v_{i}^{2}\right)^{-1}, w_{i}=w_{0} v_{i}^{2}$, $i=1, \ldots, n$. Set the iterations counter $k=1$ and go to the next step.

- Step $2 b$ : Set $w=\left[w_{1}, \ldots, w_{n}\right]^{T}$ and compute

$$
\begin{aligned}
& E=\sqrt{\frac{\hat{P}_{x x}^{k-1 \mid k-1}}{w_{0}}}\left(I+v v^{T}\right)^{-\frac{1}{2}} \operatorname{diag}(v)^{-1}, e=\frac{-E w}{w_{0}}, \\
& \chi_{i}=\hat{x}_{k-1 \mid k-1}+([E, e])_{* i}, \quad i=0, \ldots, n .
\end{aligned}
$$

Go to Step 3 and follow it completely, but with its last command referring to Step $2 b$ instead of Step 2.

Remark 2.1: The strategy in the Additive Homogeneous Symmetric Minimum UKF requires the evaluation of $2 n+1$ sigma points at each iteration, whereas the two others (i.e., Additive Rho Minimum UKF and Additive General Minimum $\mathrm{UKF}$ ) require $n+1$. Apart from the computational effort, all of the UKF algorithms presented an appreciable performance for estimating the position of an automotive electronic throttle valve, as detailed in the next section.

\section{Automotive Electronic throttle VAlVe}

Even though successful for many instances, modeling the throttle remains a challenge since (i) its assemblage is not unique and (ii) the throttle has nonlinear dynamics due to the stick-slip, hysteresis, restoring springs, and limp-home constraints [2], [7], [13], [30], [31]. Our approach contributes towards the modeling and estimation of such nonlinear device, as detailed next.

The experiments presented in this section were conducted in a laboratory testbed with the following equipments: a unity of Quanser Q4 Real-Time Control Board that allowed us to communicate real-time data with Matlab-Simulink software; a unity of Quanser UPM180-25-B-PWM Power Amplifier to supply the voltage and electrical current consumed by the equipments; and a unity of the automotive electronic throttle body made up by Continental Siemens VDO, Model A2C59511705, P.N. 06F133062J. The acquisition card of the Quanser Q4 Board was configured to work with data sampling fixed at $1 \mathrm{~ms}$.

The throttle is assembled with an internal sensor of position, which maps the range of operation from zero to ninety degrees into zero to five Volts, in a linear relationship. The velocity of the valve can be computed by a numerical approximation of the derivative of position. The electric power consumed by the throttle was measured by a wattmeter.

\section{A. Modeling}

According to [6] and [32], the throttle can be modeled as a piecewise linear system. An advantage of this piecewise setup is that it conveys the simplicity of linear systems to represent the throttle, a nonlinear device. A collateral effect is that of neglecting some significant nonlinear characteristics. Thus it seems reasonable to join these two setups into a single one, i.e., both piecewise linear dynamics [6], [32] and nonlinear dynamics [2], [7], [13], [30], [31] into a single model.

The automotive electronic throttle body is usually represented by a three-dimensional system [7], [13], [14], [33]; the three states of the system are (i) the angular position of the throttle valve $\theta$, (ii) the angular velocity of the throttle valve $\varrho$, and (iii) the electrical current consumed by the throttle $i$. The voltage applied in the terminals of the throttle represents the input of the model (i.e., $u$ ), as in the scheme shown in Fig. 1.

The model used here is based on the physically driven, traditional continuous-time model (e.g. [13, Eq. (6)], [7, Eq. (6)], [14, Eq. (8)])

$$
\begin{aligned}
\frac{\mathrm{d}}{\mathrm{d} t}\left[\begin{array}{c}
\theta_{k} \\
\varrho_{k} \\
i_{k}
\end{array}\right]=\left[\begin{array}{ccc}
0 & a_{12} & 0 \\
a_{21} & a_{22} & a_{23} \\
0 & a_{32} & a_{33}
\end{array}\right] & {\left[\begin{array}{l}
\theta_{k} \\
\varrho_{k} \\
i_{k}
\end{array}\right]+\left[\begin{array}{l}
0 \\
0 \\
b
\end{array}\right] u_{t} } \\
& +\left[\begin{array}{c}
0 \\
\varphi\left(\theta_{k}, \varrho_{k}\right) \\
0
\end{array}\right],
\end{aligned}
$$

where $\varphi: \mathbb{R}^{2} \mapsto \mathbb{R}$ denotes a piecewise linear function. Each paper [7], [13], [14] proposes a distinct format for the function $\varphi(\cdot)$, so that there is no general consensus on $\varphi(\cdot)$.

Interestingly, experimental data indicated that the nonlinearities of the throttle are more noticeable when the position of the throttle valve is near to the closed position; the effects of nonlinearities decrease as long as the valve opens. This motivated us to split the region of operation of the throttle in three main regions, aiming for improving the throttle's nonlinear representation: $\Theta_{1}=\left[0^{\circ}, 8^{\circ}\right], \Theta_{2}=\left(8^{\circ}, 16^{\circ}\right]$, and $\Theta_{3}=\left(16^{\circ}, 90^{\circ}\right]$.

Under these three regions, we considered the discrete-time version of (2); namely, with $x_{k} \equiv\left[\begin{array}{llll}0.1 \times \theta_{k} & \varrho_{k} & i_{k}\end{array}\right]^{\prime} \in \mathbb{R}^{3}$, we applied the usual Euler discretization in (2) to obtain

$$
\begin{aligned}
& x_{k+1}= {\left[\begin{array}{ccc}
1 & a_{12}^{(s)} & 0 \\
a_{21}^{(s)} & a_{22}^{(s)} & a_{23}^{(s)} \\
0 & a_{32}^{(s)} & a_{33}^{(s)}
\end{array}\right] x_{k}+\left[\begin{array}{c}
0 \\
0 \\
b^{(s)}
\end{array}\right] u_{k}+F w_{k} } \\
&+ {\left[\begin{array}{c}
c_{1}^{(s)} \operatorname{sgn}\left(\varrho_{k}\right)+c_{2}^{(s)} \operatorname{sgn}\left(\theta_{k}-1\right)+c_{3}^{(s)} \\
0
\end{array}\right], } \\
& \theta_{k} \in \Theta_{s}, \quad s=1,2,3, \quad \forall k \geq 0,
\end{aligned}
$$

where the values of $a_{12}^{(s)}, \ldots, a_{33}^{(s)}, b^{(s)}, c_{1}^{(s)}, \ldots, c_{3}^{(s)}, s=$ $1,2,3$, are available in Table I; these values were identified according to a procedure described later. For the moment, notice in (3) that the $s$-th mode is active at the $k$-th stage when $\theta_{k}$ belongs to the set $\Theta_{s}$.

\section{B. Identification}

Persistent excitation signals were applied in $u_{k}$, and the corresponding real-time system state $x_{k}$ was measured and 

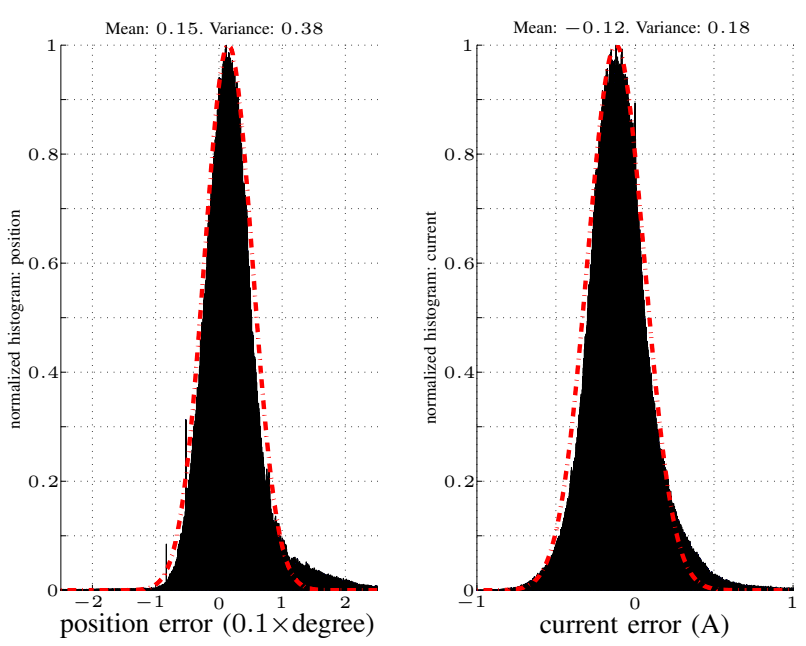

Fig. 2. Automotive electronic throttle device: normalized histogram showing the error between the model and real-time data. The picture in the left (right) shows the error for the position (electrical current) of the throttle. The histograms tend to follow Gaussian functions with null mean and variance as indicated.

stored. An amount of 3.8 million points were used in $u_{k}$, and they were carefully chosen so as to excite all the possible input-output relations for the throttle. Indeed, the values of $u_{k}$ were obtained by passing a train of pseudo-random rectangular pulses, with time-varying random amplitudes (from 0 to 10 Volts), through a fourth-order Butterworth low-pass filter with a cutoff frequency chosen randomly between 0.01 and $60 \mathrm{~Hz}$.

The parameters of (3) were chosen so as to minimize the mean square error between part of the collected data and the simulated data from (3) (with $w_{k} \equiv 0$ ). In this procedure, we used three blocks of data, and each block contained inputoutput data with ten thousand points generated via persistent excitation signals plus a DC offset.

After obtaining the parameters of (3) (cf. Table I), we checked the statistical properties of the term $w_{k}$, as follows. First, we calculated the error $e_{k}=x_{k}-\tilde{x}_{k}$, where $x_{k}$ satisfies (3) with $w_{k} \equiv 0$ and $\tilde{x}_{k}$ represents the corresponding real-time measured point; in this evaluation, we used all the previously stored 3.8 million points. Second, based on the calculated error, we made a statistical analysis (see Fig. 2 for a pictorial illustration), which suggested that $\left\{w_{k}\right\}$ is a Gaussian stationary process, and that $F$ in (3) is

$$
F=\operatorname{diag}(\sqrt{0.35}, 0, \sqrt{0.18}) .
$$

A minor bias was detected in $e_{k}$ with mean error of $1.5^{\circ}$ for angular position and $-0.12 \mathrm{~A}$ for electrical current (see Fig. 2). Although the error bias was not represented in the model (3), it was accounted appropriately in the estimation procedure, the main experimental part of this paper, to be detailed next.

\section{Case study: the automotive electronic throttle valve with no sensor of position}

As previously discussed in Introduction, a failure in the sensor of position is undesirable because it increases the risks of damage. To mitigate the effects of an eventual failure in the sensor of position, we suggest the use of unscented Kalman filters accompanied by an additional sensor, detached from the throttle's structure but connected to it electronically, as shown in Fig. 1. Showing the usefulness of this simple strategy represents the main contribution of this paper.

To clarify our main contribution, we assume hereafter that the sensor of position is damaged. To circumvent this problem, we use a wattmeter in the circuitry of the throttle, as depicted in Fig. 1.

Remark 3.1: Any instrument generating measurements that depend on the current $i_{k}$ could be used in place of a wattmeter. For instance, the wattmeter reads the power consumption $i_{k}^{2}$ plus some imprecision $v_{k}$, i.e.,

$$
y_{k}=i_{k}^{2}+v_{k}, \quad \forall k \geq 0,
$$

where $\left\{v_{k}\right\}$ represents a standard Gaussian stationary noise. With $h(\cdot)$ being any continuous function, instruments giving measurements in the form $y_{k}=h\left(i_{k}\right)+v_{k}$ could be considered in place of (4). In our experiments, the wattmeter was the chosen sensor due to its low-cost.

The value of measurements $y_{k}$ fed the unscented Kalman filters, which produce $\hat{\theta}_{k}$, an estimation of the position $\theta_{k}$. Generating $\hat{\theta}_{k}$ in practice for the automotive throttle device reinforces the contribution of this paper.

Recall the three versions of unscented Kalman filters introduced in Section II:

1) Additive Homogeneous Symmetric Minimum UKF (Steps 1a-3a).

2) Additive Rho Minimum UKF (Steps $1 a-3 a$ ).

3) Additive General Minimum UKF (Steps $1 b-3 b$ ).

These three filters were evaluated in simulation and experiments with $n=3, \lambda=12, \hat{x}_{0 \mid 0}=\left[\begin{array}{lll}0 & 0 & 0\end{array}\right]^{\prime}$, and $\hat{P}_{x x}^{0 \mid 0}=I$, as follows.

- (Simulation). Two million points were considered in the input $u_{k}$. Then these points were used in (3) to compute both the statistical mean of (3), say $\bar{x}_{k}$, and the estimation value from Steps 1-3, say $\hat{x}_{k}$. The position error is obtained by extracting the first element from the computed vectors to obtain $e_{k}=\hat{\theta}_{k}-\bar{\theta}_{k}$.

- (Practice). The same input $u_{k}$ used in the Simulation was also used in the laboratory testbed to generate $\tilde{y}_{k}$,

TABLE I

PARAMETERS OF THE NONLINEAR STOCHASTIC MODEL REPRESENTING AN AUTOMOTIVE THROTTLE BODY.

\begin{tabular}{cccc}
\hline Parameter & $s=1$ & $s=2$ & $s=3$ \\
\hline$a_{12}^{(s)}$ & -0.003 & 0.0021 & 0.0442 \\
$a_{21}^{(s)}$ & 0.148 & -0.143 & -0.0192 \\
$a_{22}^{(s)}$ & 0.9625 & 0.9941 & 0.7981 \\
$a_{23}^{(s)}$ & -0.8673 & 1.8944 & 0.3538 \\
$a_{32}^{(s)}$ & 0.0005 & -0.0004 & 0.0349 \\
$a_{33}^{(s)}$ & 0.944 & 0.9514 & 0.9043 \\
$b^{(s)}$ & 0.0741 & 0.0346 & 0.0442 \\
$c_{1}^{(s)}$ & -0.0654 & -0.1068 & -0.0055 \\
$c_{2}^{(s)}$ & -0.007 & 0.0529 & 0.0615 \\
$c_{3}^{(s)}$ & 0.2255 & -0.3419 & -0.0862 \\
\hline
\end{tabular}




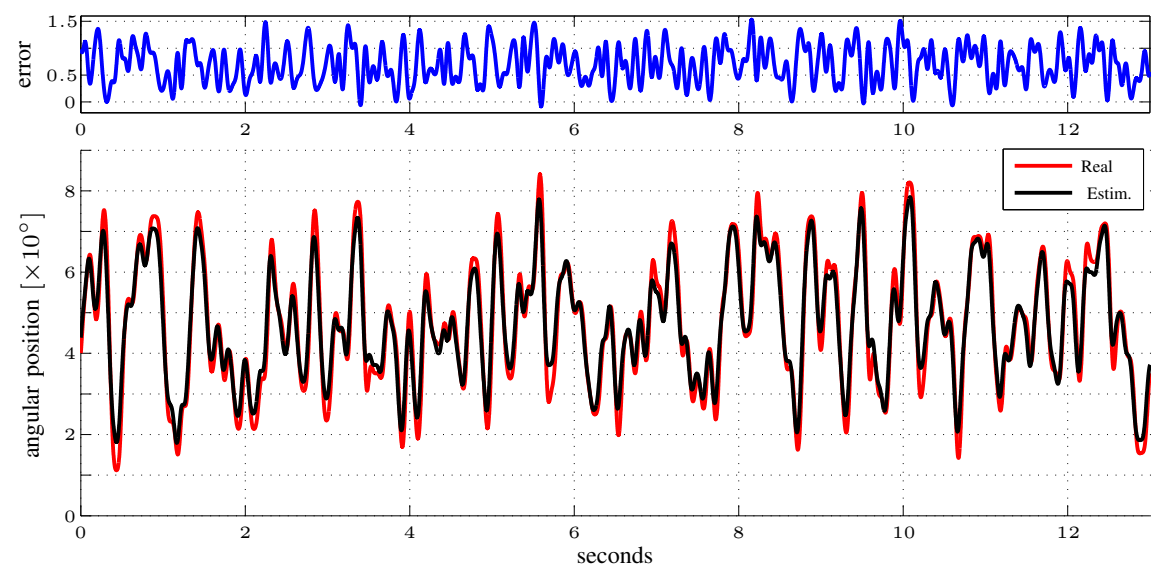

Fig. 3. Real-time position (measured) and estimated position for an automotive throttle device. The estimated position was calculated by an unscented Kalman filter, which was fed only with measurements of the electrical power consumed by the throttle.

which denotes the value collected from the wattmeter in practice. Both $u_{k}$ and $\tilde{y}_{k}$ were applied in the algorithm of Steps 1-3 to generate an estimation of the system state, say $\tilde{x}_{k}^{\text {est }}$. The first element of the vector $\tilde{x}_{k}^{\text {est }}$ is $\tilde{\theta}_{k}^{\text {est }}$, the estimated position. The sensor of position was used to generate $\tilde{\theta}_{k}$, the real value of the position of the throttle. Finally, the error produced by the estimation procedure was computed in $e_{k}=\tilde{\theta}_{k}^{\text {est }}-\tilde{\theta}_{k}$.

Table II presents the values of the mean and standard deviation of the error for the three filters for both cases, simulation and practice. As expected, the error in the simulation is smaller than the one observed in practice.

Besides, a conclusion drawn from Table II is that all filters produced a practical error of around $2.2^{\circ} \pm 13.4^{\circ}$ with a confidence interval of $95 \%$ (c.f. [34, Sec. D3, p. 553]). This signifies that the filters recovered the information of the position in practice with a precision close to $2.2^{\circ} \pm 13.4^{\circ}$. Subtracting the result by the bias error of $1.5^{\circ}$ observed in the model (see Section III-B), we can adjust the estimation to the improved value $0.7^{\circ} \pm 13.4^{\circ}$. These findings reinforce the contribution of this paper.

For sake of a final illustration, part of the data is depicted in Fig. 3. As can be seen, the estimated position recovered the real position within the prescribed accuracy (i.e. $0.7^{\circ} \pm 13.4^{\circ}$ ).

\section{CONCLUding REMARKS}

Our findings have practical implications, with special interest to automotive electronic throttle devices. The throttle

TABLE II

MEASURE OF THE MEAN AND STANDARD DEVIATION OF THE ERROR PRODUCED BY UNSCENTED KALMAN FILTERS WHEN THEY WERE USED TO ESTIMATE THE POSITION OF AN AUTOMOTIVE THROTTLE BODY.

\begin{tabular}{llcccc}
\hline & \multicolumn{2}{c}{ Simulation } & \multicolumn{2}{c}{ Experiment } \\
\hline \multicolumn{2}{c}{ UKF Filters } & Mean $\left(^{\circ}\right)$ & Std $\left(^{\circ}\right)$ & Mean $\left(^{\circ}\right)$ & Std $\left(^{\circ}\right)$ \\
\hline AHSM & Steps $1-3$ & -0.090 & 4.002 & 2.206 & 6.749 \\
ARM & Steps 1 $-3 a$ & -0.071 & 4.061 & 2.225 & 6.696 \\
AGM & Steps $1 b-3 b$ & -0.078 & 3.983 & 2.219 & 6.560 \\
\hline
\end{tabular}

device has a unique sensor that measures the angular position of the throttle's valve, and for this reason failures in this solitary sensor increase the risks of damage on the underlying devices. Wishing to mitigate the impact of a failure from the sensor of position, we suggest an approach that joins unscented Kalman filters with measurements produced by a wattmeter.

The novelty here relies on the use of a wattmeter to measure the electric power consumed by the throttle. As detailed in Remark 3.1, the wattmeter was preferred due to its low cost. However, any other kind of instruments could be used in place of a wattmeter without necessity of modifying the technique proposed in this paper.

Measurements from the wattmeter fed the unscented Kalman filter, and the filter, in its turn, generated estimation for the position of the throttle. To the best of the authors' knowledge, this paper is the first to combine estimation with an external sensor, aiming to improve the throttle's functionality.

Experiments that were carried out in laboratory showed promising results - the experimental data suggested an error of $0.7^{\circ} \pm 13.4^{\circ}$ (confidence level of $95 \%$ ) for the estimated position. This finding was quite accurate, since the estimation was taken over a range from $0^{\circ}$ to $90^{\circ}$. This evidence corroborates the novelty of our approach.

\section{REFERENCES}

[1] R. Conatser, J. Wagner, S. Ganta, and I. Walker, "Diagnosis of automotive electronic throttle control systems," Control Engineering Practice, vol. 12, no. 1, pp. 23 - 30, 2004.

[2] J. Deur, D. Pavković, N. Perić, M. Jansz, and D. Hrovat, "An electronic throttle control strategy including compensation of friction and limphome effects," IEEE Trans. Industry Appl., vol. 40, no. 3, pp. 821-834, 2004.

[3] M. di Bernardo, A. di Gaeta, U. Montanaro, J. M. Olm, and S. Santini, "Experimental validation of the discrete-time MCS adaptive strategy," Control Engineering Practice, vol. 21, no. 6, pp. 847-859, 2013.

[4] M. di Bernardo, A. di Gaeta, U.Montanaro, and S. Santini, "Synthesis and experimental validation of the novel LQ-NEMCSI adaptive strategy on an electronic throttle valve," IEEE Trans. Control Syst. Technol., vol. 18 , no. 6, pp. $1325-1337,2010$.

[5] R. Grepl and B. Lee, "Modeling, parameter estimation and nonlinear control of automotive electronic throttle using a rapid-control prototyping technique," International Journal of Automotive Technology, vol. 11, no. 4 , pp. 601-610, 2010. 
[6] M. Vasak, M. Baotic, I. Petrovic, and N. Peric, "Hybrid theory-based time-optimal control of an electronic throttle," IEEE Trans. Industrial Electronics, vol. 54, no. 3, pp. 1483 -1494, 2007.

[7] Y. Pan, U. Ozguner, and O. H. Dagci, "Variable-structure control of electronic throttle valve," IEEE Trans. Industrial Electronics, vol. 55, no. 11 , pp. 3899-3907, 2008.

[8] U. Montanaro, A. di Gaeta, and V. Giglio, "Robust discrete-time MRAC with minimal controller synthesis of an electronic throttle body," IEEE/ASME Transactions on Mechatronics, vol. 19, no. 2, pp. 524-537, 2014.

[9] M. Reichhartinger and M. Horn, "Application of higher order slidingmode concepts to a throttle actuator for gasoline engines," IEEE Trans. Industrial Electronics, vol. 56, no. 9, pp. 3322 -3329, 2009.

[10] J. Finch, "Toyota sudden acceleration: A case study of the national highway traffic safety administration-recalls for change," Loyola Consumer Law Review, vol. 22, no. 472-496, pp. 4-9, 2010, available at www.lawecommons.luc.edu/lclr/vol22/iss $4 / 4$.

[11] E. George and M. Pecht, "Tin whisker analysis of an automotive engine control unit," Microelectronics Reliability, vol. 54, no. 1, pp. 214-219, 2014.

[12] B. Sood, M. Osterman, and M. Pecht, "Tin whisker analysis of Toyota's electronic throttle controls," Circuit World, vol. 37, no. 3, pp. 4-9, 2011.

[13] X. Yuan, Y. Wang, and L. Wu, "SVM-based approximate model control for electronic throttle valve," IEEE Trans. Vehicular Technology, vol. 57, no. 5, pp. 2747-2756, 2008.

[14] X. Jiao, J. Zhang, and T. Shen, "An adaptive servo control strategy for automotive electronic throttle and experimental validation," IEEE Transactions on Industrial Electronics, vol. 61, no. 11, pp. 6275-6284, 2014.

[15] S. J. Julier, J. K. Uhlmann, and H. F. Durrant-Whyte, "A new approach for filtering nonlinear systems," in Proc. IEEE American Control Conf. (ACC'95), Seatle, WA, 1995, pp. 1628-1632.

[16] S. J. Julier and J. K. Uhlmann, "Consistent debiased method for converting between polar and Cartesian coordinate systems," in Proc. SPIE, vol. 3086, 1997, pp. 110-121.

[17] - "A New Extension of the Kalman Filter to Nonlinear Systems." in Proc. SPIE AeroSense, vol. 3068, 1997, pp. 182-193.

[18] I. J. Y. Menegaz H. M. and B. G. A., "New minimum sigma set for unscented filtering," Int. J. Robust. Nonlinear Control, 2014, doi: $10.1002 /$ rnc. 3262.

[19] H. M. Menegaz, J. Y. Ishihara, G. A. Borges, and A. N. Vargas, "A systematization of the unscented Kalman filter theory," IEEE Trans. Automat. Control, vol. 60, pp. 2583-2598, 2015.

[20] R. Kandepu, B. Foss, and L. Imsland, "Applying the unscented Kalman filter for nonlinear state estimation," Journal of Process Control, vol. 18, no. 7-8, pp. 753-768, 2008.

[21] P. Stano, Z. Lendek, J. Braaksma, R. Babuska, C. de Keizer, and A. J. den Dekker, "Parametric Bayesian filters for nonlinear stochastic dynamical systems: A survey," Cybernetics, IEEE Trans., vol. 43, no. 6, pp. 1607-1624, 2013.

[22] K. Xiong, H. Y. Zhang, and C. W. Chan, "Performance evaluation of UKF-based nonlinear filtering," Automatica, vol. 42, no. 2, pp. 261-270, 2006.

[23] J. Walters-Williams and Y. Li, "Comparison of extended and unscented Kalman Filters applied to EEG signals," in Proc. IEEE/ICME Int. Conf. Complex Medical Eng. (ICCME'10), Gold Coast, QLD, 2010, pp. 4551.

[24] C. Eberle and C. Ament, "The Unscented Kalman Filter estimates the plasma insulin from glucose measurement." BioSystems, vol. 103, no. 1, pp. 67-72, 2011.

[25] J. Zhang and C. Xia, "State-of-charge estimation of valve regulated lead acid battery based on multi-state Unscented Kalman Filter," Int. J. Elect. Power Energy Syst., vol. 33, no. 3, pp. 472-476, Mar. 2011.

[26] B. O. S. Teixeira, L. A. B. Tôrres, P. Iscold, and L. A. Aguirre, "Flight path reconstruction - A comparison of nonlinear Kalman filter and smoother algorithms," Aerospace Science and Technology, vol. 15, no. 1, pp. 60-71, 2011.

[27] G. Chowdhary and R. Jategaonkar, "Aerodynamic parameter estimation from flight data applying extended and unscented Kalman filter," Aerospace Science and Technology, vol. 14, no. 2, pp. 106-117, 2010.

[28] S. Holmes, G. Klein, and D. W. Murray, "An O(N(2)) Square Root Unscented Kalman Filter for Visual Simultaneous Localization and Mapping," IEEE Trans. Pattern Anal. Mach. Intell., vol. 31, no. 7, pp. 1251-63, 2009.

[29] H. M. Menegaz, J. Y. Ishihara, and G. A. Borges, "A new smallest sigma set for the Unscented Transform and its applications on SLAM," in Proc.
IEEE Decision Control and European Control Conf. (CDC/ECC'11), Orlando, FL, 2011, pp. 3172-3177.

[30] C. Rossi, A. Tilli, and A. Tonielli, "Robust control of a throttle body for drive by wire operation of automotive engines," IEEE Trans. Control Syst. Technol., vol. 8, no. 6, pp. $993-1002,2000$.

[31] A. Thomasson and L. Eriksson, "Model-based throttle control using static compensators and pole placement," Oil \& Gas Science and Technology - Rev. IFP, vol. 66, no. 4, pp. 717-727, 2011.

[32] M. Morari, M. Baotic, and F. Borrelli, "Hybrid systems modeling and control," European Journal of Control, vol. 9, no. 2-3, pp. 177-189, 2003.

[33] X. Yuan, Y. Wang, L. Wu, X. Zhang, and W. Sun, "Neural network based self-learning control strategy for electronic throttle valve," IEEE Trans. Vehicular Technology, vol. 59, no. 8, pp. 3757-3765, 2010.

[34] E. W. Grafarend, Linear and nonlinear models: fixed effects, random effects, and mixed models. Berlin, Germany: Walter de Gruyter GmbH, 2006. 